\title{
Sliding Control of an Electropneumatic Actuator Using an Integral Switching Surface
}

\author{
Mohamed Bouri and Daniel Thomasset
}

\begin{abstract}
This paper presents a synthesis of a nonlinear switching control of a rotational electropneumatic servodrive using a sliding mode approach. A nonlinear sliding mode control law is applied to the system under consideration. First, the model of the electropneumatic servodrive is developed. This model is nonlinear with respect to both the state variables and the control input. It is transformed to be linear with respect to a new control variable and a coordinate transformation is then related to make possible the implementation of the nonlinear discontinuous controller. Two sliding mode controllers are synthesized with and without an integral term in the switching surface. The sliding regimes are particularly pointed out and their stability analyzed to show that the integral discontinuous control provides best results especially for a steady-state error cancellation. Practical considerations are proposed for choosing the control parameters and finally, the experimental results are presented and discussed.
\end{abstract}

Index Terms-Electropneumatic actuator, integral sliding surface, nonlinear robust control, sliding mode, tracking.

\section{NOMENCLATURE}

$\theta, \omega, \gamma$
$K_{c}$
$P_{x}$
$K_{\theta}$
$P_{s}, P_{e}$
$T$
$V_{x}$
$i$
$k$
$M, J$
$r$
$\operatorname{Re}$
$\Gamma_{\mathrm{dry}}$
$g$
$l$
$\phi$
$W, \lambda$
$q_{m^{p}}\left(i, P_{s}, P_{e}, P_{p}\right)$

$q_{m^{n}}\left(i, P_{s}, P_{e}, P_{n}\right)$

Position, speed, acceleration.

Torque constant.

Pressure in the chamber $x$.

Volume constant.

Supply and exhaust pressure.

Ambient temperature.

Volume of the chamber $x$.

Servovalve current.

Polytropic constant.

Load and total inertia.

Perfect gas constant.

Reynolds number.

Dry friction torque.

Gravity constant.

Mass position on the paddle.

Boundary layer thickness.

Switching gain and surface parameter. Mass flow rates provided from the servovalve to the cylinder chambers.

Manuscript received February 12, 1999; revised March 2, 2000. Recommended by Associate Editor K. Moore. This work was supported by grant of the PRC-GDR Automatic Control of the French National Centre for Scientific Research CNRS.

M. Bouri is with EPFL, Département de Microtechnique, Institut des Systèmes Robotiques, $\mathrm{CH}-1015$, Switzerland.

D. Thomasset is with INSA de Lyon, Laboratoire d'Automatique Industrielle, 69621 Villeurbonne Cedex, France.

Publisher Item Identifier S 1063-6536(01)00525-5.

$$
\begin{aligned}
& q_{m^{s x}}\left(i, P_{s}, P_{x}\right), \\
& q_{m^{x e}}\left(i, P_{x}, P_{e}\right) \\
& q_{m^{v}}\left(P_{p}, P_{n}\right) \\
& A_{x y}(i)
\end{aligned}
$$

\begin{abstract}
Mass flow rates in the half bridges " $x$ " of the servovalve, with $x=p$ or $n$.

Interchamber leakage mass flow rate. Variable restriction between $x$ and $y$.
\end{abstract}

\section{INTRODUCTION}

$\mathbf{S}$ EVERAL industries are concerned with the "fluidpower" field, namely in applications requiring an accurate control of mechanical variables (position, velocity, and force), and/or where strong forces or a large bandwidth are required and in certain environments when electrical systems are not useful.

In this paper the position tracking of a rotational electropneumatic servodrive is treated. The sliding mode control has been chosen because of its robustness vis-a-vis modeling errors and unknown terms. References [1]-[6] can be examined for detailed examples.

To control an electropneumatic actuator, one implicitly requires the measurement or estimation of a minimum of three variables: position, velocity, and acceleration. These depend on the type of model under consideration, i.e., nonlinear or linear tangent models, and also on the type of the applied control. We have seen [6] that controlled by the nonlinear input-output linearizing control, the acceleration measurement considerably improves the positioning performances and this provides better results when using a sliding mode technique. However, the accelerometer sensor is not automatically used because of its cost and its industrial unusefulness. The acceleration information is therefore replaced by the pressure difference, but this leads to a steady-state error.

To overcome this anomaly, this paper proposes an integral sliding mode control law to cancel this static error and is organized as follows.

First, the nonlinear model of the rotational electropneumatic servodrive is presented and the state-space equations governing the motion of this plant are put in a nonlinear affine form. In order to use a sliding mode technique, a coordinate transformation is then proposed and the model is rewritten in a nominal form disturbed by an uncertainty term.

Two sliding mode controllers are synthesized with and without an integral term in the sliding surface expression and the experimental results are presented and discussed.

\section{The EleCtropneumatic System}

The system under consideration (Fig. 1) is a rotational electropneumatic servodrive controlled by a five-way servovalve (Schneider PVM 067) whose equivalent technological scheme is detailed in Fig. 2. 


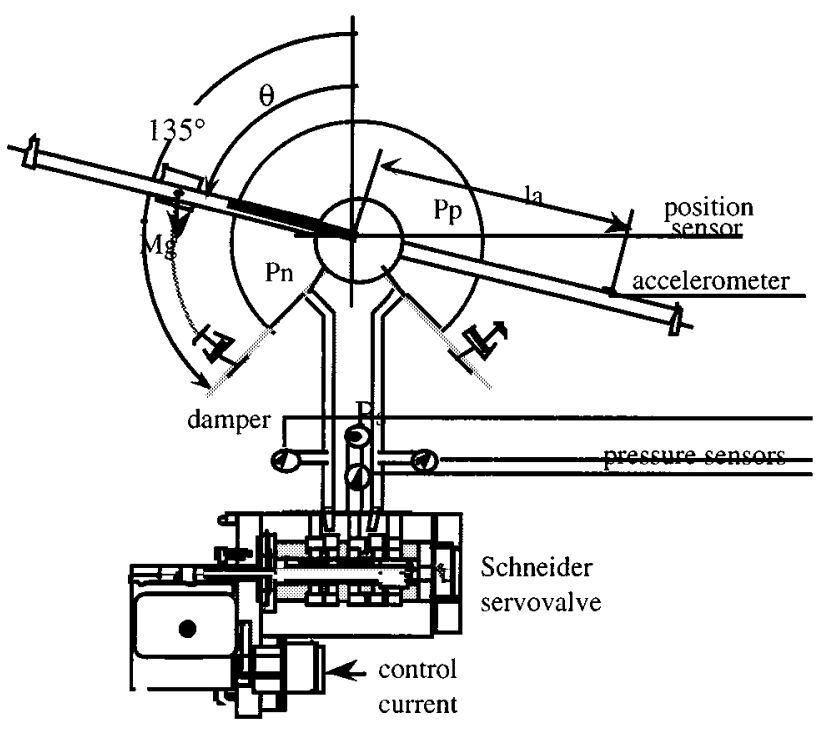

Fig. 1. The electropneumatic system.

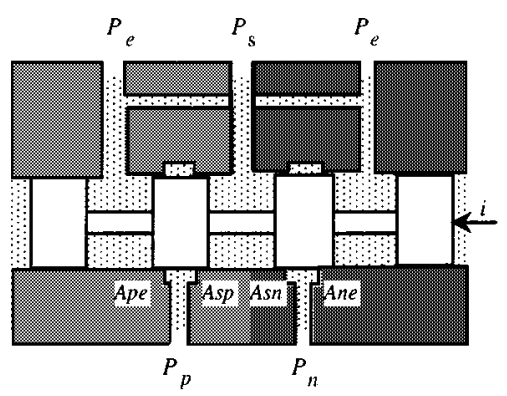

Fig. 2. Technological scheme of the servovalve.

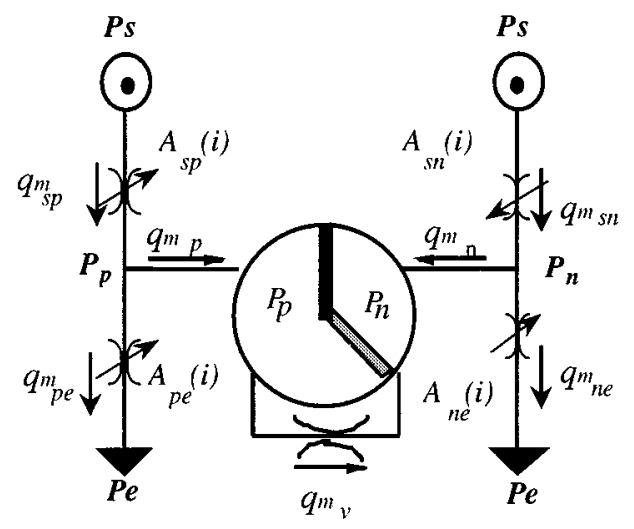

Fig. 3. Wheatstone bridge representation.

\section{A. The Nonlinear Model}

To model electropneumatic or electrohydraulic systems, the classical Wheatstone bridge representation (Fig. 3) is generally used.

As shown in Fig. 3, the electropneumatic system is composed of a servovalve and a rotational actuator. The electrical and mechanical parts of the servovalve are generally represented [7] by a third-order transfer function. Nevertheless in our case the bandwidth of the Schneider servovalve and the actuator are respectively about $160 \mathrm{~Hz}$ and $1.6 \mathrm{~Hz}$ and Bouhal [8] has shown, using the theory of multitime-scale systems, that the faster dynamics may be neglected. Therefore, the servovalve model was reduced to a static one described by two relations $q_{m^{p}}\left(i, P_{s}, P_{e}, P_{p}\right)$ and $q_{m^{n}}\left(i, P_{s}, P_{e}, P_{n}\right)$.

To obtain the actuator model two fundamental laws are used: the pressure dynamics in a chamber of variable volume and the mechanical equation.

1) The pressure evolution law in the actuator chamber is obtained by assuming the following hypotheses [9], [10].

- Air is a perfect gas and its kinetic energy is negligible.

- The pressure and the temperature in each chamber are homogeneous.

- The fluid kinetic energy within the chamber is negligible.

- The pressure evolution is polytropic.

The mass balance equation gives the pressure dynamics for the two chambers " $p$ " and " $n$ " ( $x=$ " $p$ " or " $n$ ")

$\frac{d P_{x}}{d t}=\frac{k r T_{x}}{V_{x}(\theta)}\left(\sum q_{\text {in }}-\sum q_{\text {out }}-\frac{P_{x}}{r T_{x}} \frac{d V_{x}}{d t}\right)$

with

$$
\sum q_{\mathrm{in}}=q_{m_{x}}\left(i, P_{s}, P_{e}, P_{x}\right)
$$

and

$$
\sum q_{\text {out }}=\left|q_{m^{v}}\left(P_{p}, P_{e}\right)\right|
$$

2 The principle of classic mechanics applied to the total inertia gives the following expression (viscous friction is neglected):

$$
J \frac{d \omega}{d t}=J \gamma=K_{c}\left(P_{p}-P_{n}\right)+M g l \sin (\theta)-\Gamma_{\mathrm{dry}}
$$

Assuming that the temperature variation is negligible with respect to the mean value and equal to the supply temperature (i.e., $T_{p}=T_{n}=T_{s}$ ), the electropneumatic system model is easily obtained by combining all the previous relations

$$
\begin{aligned}
\frac{d P_{p}}{d t}= & \frac{k r T_{s}}{V_{p}(\theta)}\left[q_{m^{p}}\left(i, P_{p}, P_{s}, P_{e}\right)-q_{m^{v}}\left(P_{p}, P_{n}\right)\right. \\
& \left.-\frac{K_{\theta}}{r T_{s}} P_{p} \omega\right] \\
\frac{d P_{n}}{d t}= & \frac{k r T_{s}}{V_{n}(\theta)}\left[q_{m^{n}}\left(i, P_{n}, P_{s}, P_{e}\right)-q_{m^{v}}\left(P_{p}, P_{n}\right)\right. \\
& \left.+\frac{K_{\theta}}{r T_{s}} P_{n} \omega\right] \\
\frac{d \omega}{d t}= & \frac{1}{J}\left[K_{c}\left(P_{p}-P_{n}\right)+M g l \sin (\theta)-\Gamma_{\mathrm{dry}}\right] \\
\frac{d \theta}{d t}= & \omega
\end{aligned}
$$

where $V_{p}(\theta)=V_{p o}+K_{\theta} \theta$ and $V_{n}(\theta)=V_{n o}-K_{\theta} \theta$ with $V_{p o}$ and $V_{n o}$ are the piping volumes of the chambers for the zero central position. 


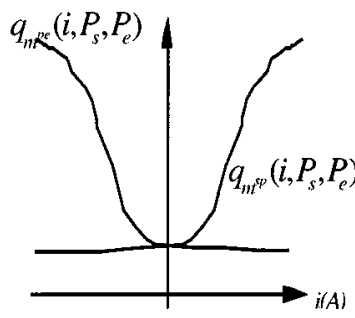

(a)

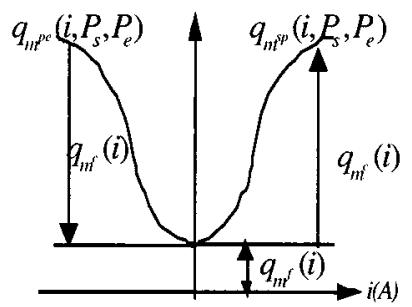

(b)
Fig. 4. (a) Real and (b) idealized mass flow-rates.

\section{B. Transformation to a Nonlinear Affine Model}

The main difficulty in the model (3) is the knowledge of the mass flow rate expressions $q_{m^{p}}(\cdot)$ and $q_{m^{n}}(\cdot)$. Two experimental methods are generally used: the first one uses a local characterization of the servovalve orifice openings [11], [12], the second proposes a global characterization [13]. Nevertheless, these two methods do not lead directly to the mathematical expressions of the mass flow rates, but lead to numerical tables usually used in simulation but not in a control model.

For the Schneider servovalve, we use the solution proposed by Richard [14]. This procedure consists of introducing a new control variable $q_{m^{c}}$ and the servovalve input current is obtained by inverting the bijective characteristic $q_{m^{c}}(i)$. This solution is summarized as follows.

- First, consider the half Wheatstone bridge " $p$ " of the servovalve (Fig. 3). For any value of the control current, the real mass flow-rates $q_{m^{s p}}\left(i, P_{s}, P_{e}\right)$ and [Fig. 4(a)] are then idealized [Fig. 4(b)] assuming the leakage mass flow-rate $q_{m^{f}}\left(P_{s}, P_{e}\right)$ independent of the pressure $P_{p}$.

The mass flow-rate variable $q_{m^{c}}$, independent of the pressure $P_{p}$, is then introduced

$q_{m^{c}}(i)=\left\{\begin{array}{cl}q_{m^{s p}}\left(i, P_{s}, P_{e}\right)-q_{m f}\left(P_{s}, P_{e}\right) & \text { if } i>0 \\ -\left(q_{m^{p e}}\left(i, P_{s}, P_{e}\right)-q_{m^{f}}\left(P_{s}, P_{e}\right)\right) & \text { if } i \leq 0 .\end{array}\right.$

- On the other hand, in the half part " $p$ " of the Wheatstone bridge (Fig. 3), we have

$q_{m^{p}}\left(i, P_{p}, P_{s}, P_{e}\right)=q_{m^{s p}}\left(i, P_{s}, P_{p}\right)-q_{m^{p e}}\left(i, P_{p}, P_{e}\right)$.

Using (4) the mass flow-rate $q_{m}$ is rewritten as follows:

$$
\begin{aligned}
& q_{m^{p}}\left(i, P_{s}, P_{e}, P_{p}\right) \\
& =\left\{\begin{array}{l}
\frac{q_{m^{s p}}\left(i, P_{s}, P_{p}\right)}{q_{m^{s p}}\left(i, P_{s}, P_{e}\right)} \cdot q_{m^{c}}(i) \\
\frac{q_{m^{p e}}\left(i, P_{p}, P_{e}\right)}{q_{m^{p e}}\left(i, P_{s}, P_{e}\right)} \cdot q_{m^{c}}(i)
\end{array}\right. \\
& +\left\{\begin{array}{l}
{\left[\frac{q_{m^{s p}}\left(i, P_{s}, P_{p}\right)}{q_{m^{s p}}\left(i, P_{s}, P_{e}\right)}-\frac{q_{m^{p e}}\left(i, P_{p}, P_{e}\right)}{q_{m^{p e}}\left(i, P_{s}, P_{e}\right)}\right] \cdot q_{m^{f}}\left(P_{s}, P_{e}\right)} \\
\text { if } i, 0 \\
{\left[\frac{q_{m^{s p}}\left(i, P_{s}, P_{p}\right)}{q_{m^{s p}}\left(i, P_{s}, P_{e}\right)}-\frac{q_{m^{p e}}\left(i, P_{p}, P_{e}\right)}{q_{m^{p e}}\left(i, P_{s}, P_{e}\right)}\right] \cdot q_{m^{f}}\left(P_{s}, P_{e}\right)}
\end{array}\right.
\end{aligned}
$$

The same decomposition is carried out for the half bridge $n$." Assuming the servovalve symmetry, $q_{m^{n}}$ is expressed as

$$
\begin{aligned}
& q_{m^{n}}\left(i, P_{s}, P_{e}, P_{n}\right) \\
& =\left\{\begin{array}{l}
-\frac{q_{m^{s n}}\left(i, P_{s}, P_{n}\right)}{q_{m^{s n}}\left(i, P_{s}, P_{e}\right)} \cdot q_{m^{c}}(i) \\
-\frac{q_{m^{n e}}\left(i, P_{n}, P_{e}\right)}{q_{m^{n e}}\left(i, P_{s}, P_{e}\right)} \cdot q_{m^{c}}(i)
\end{array}\right. \\
& +\left\{\begin{array}{l}
{\left[\frac{q_{m^{s n}}\left(i, P_{s}, P_{n}\right)}{q_{m^{s n}}\left(i, P_{s}, P_{e}\right)}-\frac{q_{m^{n e}}\left(i, P_{n}, P_{e}\right)}{q_{m^{n e}}\left(i, P_{s}, P_{e}\right)}\right] \cdot q_{m}\left(P_{s}, P_{e}\right)} \\
\text { if } i, 0 \\
{\left[\frac{q_{m^{s n}}\left(i, P_{s}, P_{n}\right)}{q_{m^{s n}}\left(i, P_{s}, P_{e}\right)}-\frac{q_{m^{n e}}\left(i, P_{n}, P_{e}\right)}{q_{m^{n e}}\left(i, P_{s}, P_{e}\right)}\right] \cdot q_{m}\left(P_{s}, P_{e}\right)}
\end{array}\right.
\end{aligned}
$$

Finally, assuming the supply pressure $P_{s}$ and the exhaust pressure $P_{e}$ constants, we express the mass flow-rate $q_{m} p$ and $q_{m^{n}}$ as follows:

$q_{m^{p}}\left(i, P_{s}, P_{e}, P_{p}\right)=-q_{m^{f p}}\left(P_{p}\right)+\varphi_{p}\left(P_{p}, \operatorname{sign}\left(q_{m^{c}}\right)\right) q_{m^{c}}(i)$
$q_{m^{n}}\left(i, P_{s}, P_{e}, P_{n}\right)=-q_{m^{f n}}\left(P_{n}\right)+\varphi_{n}\left(P_{n}, \operatorname{sign}\left(q_{m^{c}}\right)\right) q_{m^{c}}(i)$

with

$$
\begin{aligned}
& \varphi_{p}\left(P_{p}, \operatorname{sign}\left(q_{m^{c}}\right)\right) \\
& = \begin{cases}\varphi_{1}\left(P_{p}\right)=\frac{q_{m^{s p}}\left(i^{*}, P_{s}, P_{p}\right)}{q_{m^{s p}}\left(i^{*}, P_{s}, P_{e}\right)} & \text { if } q_{m^{c}}>0 \\
\varphi_{2}\left(P_{p}\right)=\frac{q_{m^{p e}}\left(i^{*}, P_{p}, P_{e}\right)}{q_{m p e}\left(i^{*}, P_{s}, P_{e}\right)} & \text { if } q_{m^{c}} \leq 0\end{cases} \\
& \varphi_{n}\left(P_{n}, \operatorname{sign}\left(q_{m^{c}}\right)\right) \\
& = \begin{cases}\varphi_{1}\left(P_{n}\right)=\frac{q_{m^{n e}}\left(i^{*}, P_{n}, P_{e}\right)}{q_{m^{n e}}\left(i^{*}, P_{s}, P_{e}\right)} & \text { if } q_{m^{c}}>0 \\
\varphi_{2}\left(P_{n}\right)=\frac{q_{m^{s n}}\left(i^{*}, P_{s}, P_{n}\right)}{q_{m^{s n}}\left(i^{*}, P_{s}, P_{e}\right)} & \text { if } q_{m^{c}} \leq 0 .\end{cases}
\end{aligned}
$$

and

$$
\begin{aligned}
q_{m f p}\left(P_{p}\right) & =q_{m^{f}}\left(P_{s}, P_{e}\right)\left(\varphi_{1}\left(P_{p}\right)-\varphi_{2}\left(P_{p}\right)\right) \\
q_{m^{f n}}\left(P_{n}\right) & =q_{m} f\left(P_{s}, P_{e}\right)\left(\varphi_{1}\left(P_{n}\right)-\varphi_{2}\left(P_{n}\right)\right)
\end{aligned}
$$

( $i^{*}$ denotes the used value of the current in the measurements).

The experimental values of the $\varphi$ functions and their polynomial fits are shown in Fig. 5.

The measured leakages in the servovalve and between the chambers $(p)$ and $(n)$ are given, respectively, by the curves in Fig. 6 (polynomial fits are computed)

The nonlinear affine model is then given by

$$
\begin{aligned}
& \dot{x}=f(x)+g(x) u \\
& y=h(x)=\theta
\end{aligned}
$$

$$
f(x)=\left(\begin{array}{c}
\frac{k r T}{V_{p}(\theta)}\left[-q_{m^{f p}}\left(P_{p}\right)-q_{m^{v}}\left(P_{p}, P_{n}\right)-\frac{K_{\theta}}{r T} P_{p} \omega\right] \\
\frac{k r T}{V_{n}(\theta)}\left[-q_{m^{f n}}\left(P_{n}\right)+q_{m^{v}}\left(P_{p}, P_{n}\right)+\frac{K_{\theta}}{r T} P_{n} \omega\right] \\
\frac{1}{J}\left[K_{c}\left(P_{p}-P_{n}\right)+M g l \sin (\theta)-\Gamma_{\mathrm{dry}}\right] \\
\omega
\end{array}\right)
$$




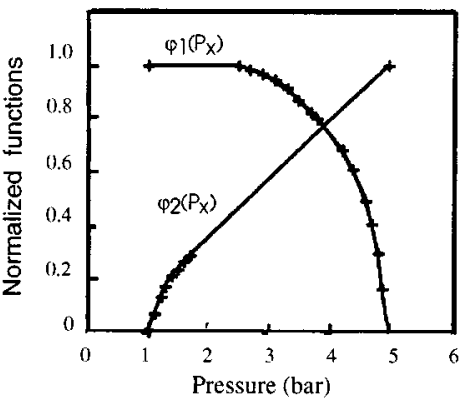

Fig. 5. Experimental mass flow-rate functions and polynomials.
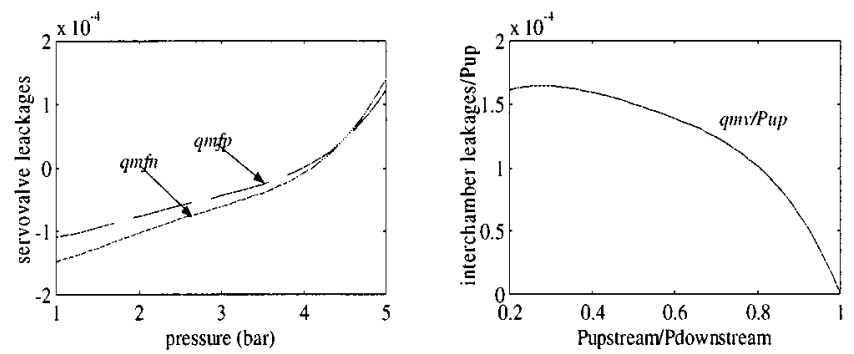

Fig. 6. Mass flow rate leakages.

and

$$
g(x)=\left(\begin{array}{cc}
\frac{k r T}{V_{p}(\theta)} \varphi_{p}\left(P_{p} \operatorname{sign}(u)\right) \\
-\frac{k r T}{V_{n}(\theta)} \varphi_{n}\left(P_{n}, \operatorname{sign}(u)\right) \\
0 \\
0 .
\end{array}\right)
$$

$x=\left(P_{p}, P_{n}, \omega, \theta\right)$ is the state vector and the mass flow rate control variable $q_{m^{c}}(i)$ is replaced by " $u$ " in order to use classical notations.

\section{CONTROL SYNTHESIS}

\section{A. Coordinate Transformation}

Let us now consider the nonlinear affine model (11) of the electropneumatic system. The output $h(x)=\theta$ has a relative degree $r=3$. The following change of coordinates is carried out:

$$
\begin{aligned}
& z_{1}=\phi_{1}(x)=\theta \\
& z_{2}=\phi_{2}(x)=\omega \\
& z_{3}=\phi_{3}(x)=\frac{K_{c}}{J}\left(P_{p}-P_{n}\right) \\
& z_{4}=\phi_{4}(x)=P_{p} .
\end{aligned}
$$

It is easy to check that it is actually a global diffeomorphism due to the property of the nonsingularity of its Jacobean matrix $\left(|\partial \Phi / \partial x|=-\left(K_{c} / J\right)\right)$.

In these new coordinates $z=\left(z_{1}, z_{2}, z_{3}, z_{4}\right)^{T}$, the system dynamics may be rewritten in the following form:

$$
\begin{aligned}
& \dot{z}_{1}=z_{2} \\
& \dot{z}_{2}=z_{3}+\delta\left(\Phi^{-1}(z)\right) \\
& \dot{z}_{3}=\alpha_{n}\left(\Phi^{-1}(z)\right)+\Delta_{f}\left(\Phi^{-1}(z)\right)+\beta_{n}\left(\Phi^{-1}(z)\right) u \\
& \dot{z}_{4}=\dot{P}_{p}
\end{aligned}
$$

$\alpha_{n}$ and $\beta_{n}$ are expressed in the $x$ coordinates as

$$
\begin{aligned}
\alpha_{n}(x)= & -\frac{k K_{c} K_{\theta}}{J} \omega\left(\frac{P_{p}}{V_{p}(\theta)}+\frac{P_{n}}{V_{n}(\theta)}\right) \\
\beta_{n}(x)= & \frac{k r T K_{c}}{J}\left(\frac{\varphi_{p}\left(P_{p}, \operatorname{sign}(u)\right)}{V_{p}(\theta)}\right. \\
& \left.+\frac{\varphi_{n}\left(P_{n}, \operatorname{sign}(u)\right)}{V_{n}(\theta)}\right) .
\end{aligned}
$$

and

$$
\begin{gathered}
\Delta_{f}=\frac{k r T K}{J}\left(\begin{array}{c}
\frac{-\dot{m}_{f p}\left(P_{p}\right)-\dot{m}_{v}\left(P_{p}, P_{n}\right)}{V_{p}(\theta)} \\
+\frac{\dot{m}_{f n}\left(P_{n}\right)-\dot{m}_{v}\left(P_{p}, P_{n}\right)}{V_{n}(\theta)}
\end{array}\right) \\
\delta=\frac{M g l}{J} \sin (\theta)-\frac{\Gamma_{\text {dry }}}{J}=A \sin (\theta)+B .
\end{gathered}
$$

In practice, this form is very useful for the following reasons.

- It contains only accessible states (position, velocity, and the two chamber pressures).

- The terms $\alpha_{n}\left(\Phi^{-1}(z)\right)$ and $\beta_{n}\left(\Phi^{-1}(z)\right)$ contain only known terms.

- All the unknown terms (dry friction, gravity torque (because $M$ may vary), servovalve and interchamber leakages) are grouped in uncertainty terms $\Delta_{f}$ and $\delta$ ( $\delta$ is an unmatched uncertainty representing the difference between the real acceleration and the state variable $z_{3}$ ).

\section{B. Implementation}

Let:

- $\theta_{d}(t), \omega_{d}(t), \gamma_{d}(t)$ and $\theta_{d}^{(3)}(t)$ be the desired position, velocity, acceleration and jerk trajectories;

- $\tilde{z}=\left(\tilde{z}_{1}, \tilde{z}_{2}, \tilde{z}_{3}\right)=\left(e_{\theta}, e_{\omega}, e_{\gamma}\right)$ be the state error vector containing the position, velocity, and acceleration errors, respectively

$$
e_{\theta}=\theta-\theta_{d}(t), \quad e_{\omega}=\omega-\omega_{d}(t), \quad e_{\gamma}=z_{3}-\gamma_{d}(t) .
$$

1) Sliding Mode Control (SMC) Implementation: Let $\sigma$ be the sliding surface (chosen in a Slotine form [16])

$$
\begin{aligned}
\sigma & =\left(\frac{d}{d t}+\lambda\right)^{r-1} \tilde{z}_{1}, \quad \lambda>0 \\
\sigma(\tilde{z}) & =\lambda^{2} \tilde{z}_{1}+2 \lambda \tilde{z}_{2}+\tilde{z}_{3}=\lambda^{2} e_{\theta}+2 \lambda e_{\omega}+e_{\gamma} .
\end{aligned}
$$

Then, the classic SMC [15], [16] law expressed in the $x$ coordinates is

$$
u=\frac{-\alpha_{n}(x)-\lambda^{2} e_{\omega}-2 \lambda e_{\gamma}+\theta_{d}^{(3)}-W \operatorname{sign}(\sigma)}{\beta_{n}(x)} .
$$

The switching gain $W$ must obey the relation (19) in order to maintain the sliding mode condition $\sigma \dot{\sigma}<0 . \delta_{\max }$ and $\Delta_{f \max }$ are the bounds of $\delta$ and $\Delta_{f}$

$$
W>\Delta_{f \max }+2 \lambda \delta_{\max } .
$$

The singularity of this control law happens when $\left(\varphi_{p}(\cdot)=\right.$ $\left.\varphi_{n}(\cdot)=0\right)$. This occurs when the pressures in the two chambers " $p$ " and " $n$ " are equal to the source and exhaust pressures, respectively. This situation is rare in practice. However, fixing the 
value of the denominator $\beta_{n}(x)$ when it is close to zero would alleviate this.

It can be easily shown that this control law $u$ is implicit due to the dependence of the denominator on the sign of $u$. However, the experimental $\varphi$ functions are positive except for the singular points defined above. $\beta_{n}(x)$ is then always positive and $\alpha_{n}(x)$ is independent of $u$. Consequently the sign of the control $u$ depends only on its numerator value.

The next step is the problem of the boundedness of the residual dynamics (14) in the sliding regime. The mathematical study of this nonlinear dynamics is difficult because of the presence of uncertainties in the nonlinear differential equation defining $P_{p}$. Nevertheless in static phase (i.e., actuator stopped, $\theta=\theta_{0}$ and $\omega=\omega_{d}(t)=0$ even if there is a steady-state error), we have proven [19] the existence, the unicity and the asymptotic stability of the equilibrium point of this system dynamics. Furthermore, in all cases, experimental results show that the two pressures are bounded and well stabilized in the final regime.

a) Sliding mode analysis: The sliding mode is given when $\sigma=0$ by the following equation:

$$
\dot{\tilde{z}}=\chi(\tilde{z})+\delta(\tilde{z}, t)=\left(\begin{array}{c}
\tilde{z}_{2} \\
-\lambda^{2} \tilde{z}_{1}-2 \lambda \tilde{z}_{2}
\end{array}\right)+\left(\begin{array}{l}
0 \\
1
\end{array}\right) \delta(\tilde{z}, t) .
$$

This time varying dynamics does not present an equilibrium state. Its total stability [17] can easily be checked. Nevertheless this result only shows that for a bounded disturbance $\delta$ the perturbed system is ultimately bounded.

In the Appendix, we prove that the error dynamics is bounded by the value $e_{\theta \max }=\delta_{\max } / \lambda^{2}$. To do a bit more then this, let us analyze what happens when the permanent phase of tracking is reached, i.e., when moving close to the ball of radius $e_{\theta \max }$. From (16), we have

$$
\begin{aligned}
\delta(\tilde{z}, t) & =A \sin \left(\tilde{z}_{1}+\theta_{d}(t)\right)+B \\
& =A \sin \left(\tilde{z}_{1}\right) \cos \left(\theta_{d}(t)\right)+A \cos \left(\tilde{z}_{1}\right) \sin \left(\theta_{d}(t)\right)+B .
\end{aligned}
$$

When $e_{\theta}$ is close to the ball defined above, $\delta(\tilde{z}, t)$ may be approximated by

$$
\begin{aligned}
\delta(\tilde{z}, t)= & A \cos \left(\theta_{d}(t)\right) \tilde{z}_{1}+A \sin \left(\theta_{d}(t)\right)+B \\
\dot{\tilde{z}}= & \left(\begin{array}{c}
\tilde{z}_{2} \\
-\lambda^{2}\left(1-\frac{A \cos \left(\theta_{d}(t)\right)}{\lambda^{2}}\right) \tilde{z}_{1}-2 \lambda \tilde{z}_{2}
\end{array}\right) \\
& +\left(\begin{array}{l}
0 \\
1
\end{array}\right)\left(A \sin \left(\theta_{d}(t)\right)+B\right) .
\end{aligned}
$$

Using physical considerations, i.e., that

$$
\frac{A}{\lambda^{2}}=\frac{M g l}{J \lambda^{2}} \ll 1 \text {. }
$$

We can once more transform the system to the following representation:

$$
\dot{\tilde{z}}=\left(\begin{array}{c}
\tilde{z}_{2} \\
-\lambda^{2} \tilde{z}_{1}-2 \lambda \tilde{z}_{2}
\end{array}\right)+\left(\begin{array}{l}
0 \\
1
\end{array}\right)\left(A \sin \left(\theta_{d}(t)\right)+B\right) .
$$

Which can be considered as a linear system with a natural frequency $\lambda$, a gain $1 / \lambda^{2}$ and a sinusoidal input with an offset.

Hence, because $\lambda \gg \omega_{d}$ (from experimental values), the position error $e_{\theta}$ tracks exactly the time varying function $\left(A \sin \left(\theta_{d}(t)\right)+B\right) / \lambda^{2}$. Furthermore, when in the static phases, i.e., $\omega_{d}=0$ (starting and breaking phases), the output of (21) surely leads to the static error $\delta_{\text {sta }} / \lambda^{2}\left(\delta_{\text {sta }}\right.$ corresponds to the static value of $\delta$ ).

Practically, as underlined above, the great advantage of the proposed control is its state feedback that does not need an accelerometer sensor. However the position error when the actuator stops is nonzero.

2) Sliding Mode Controller with an Integral surface (SMCI) Implementation: In order to cancel the static error, an integral term is added to the surface expression. Let $\sigma_{I}$ be this new surface also chosen in a Slotine form

$$
\begin{aligned}
\sigma_{I}(\tilde{z}) & =\left(\lambda+\frac{d}{d t}\right)^{3} \int_{0}^{t} \tilde{z}_{1} d \tau \\
& =\lambda^{3} \int_{0}^{t} e_{\theta} d \tau+3 \lambda^{2} e_{\theta}+3 \lambda e_{\omega}+e_{\gamma} .
\end{aligned}
$$

The corresponding SMCI is synthesized as (18) and is given by

$u_{1}=\frac{-\alpha_{n}(x)-\lambda^{3} e_{\theta}-3 \lambda^{2} e_{\omega}-3 \lambda e_{\gamma}+\theta_{d}^{(3)}-W \operatorname{sign}\left(\sigma_{1}\right)}{\beta_{n}(x)}$.

The surface attractivity is maintained by choosing $W$ such that

$$
W>\Delta_{f \max }+3 \lambda \delta_{\max }
$$

a) Sliding mode analysis: When in sliding mode the closed-loop system dynamics obey the following equation:

$$
\begin{aligned}
& \dot{\xi}_{1}=\xi_{2} \quad \text { with } \quad \xi_{1}=\int_{0}^{t} e_{\theta} d \tau \\
& \dot{\xi}_{2}=\xi_{3} \\
& \dot{\xi}_{3}=-\lambda^{3}\left(\xi_{1}-\frac{\delta}{\lambda^{3}}\right)-3 \lambda^{2} \xi_{2}-3 \lambda \xi_{3} .
\end{aligned}
$$

Like in the previous section, with the same calculus and the same physical assumptions, we easily prove that $\xi_{1}$ will track the time varying function $\left(A \sin \left(\theta_{d}(t)\right)+B\right) / \lambda^{2}$ and thus, the position error $e_{\theta}$ tracks $A \omega_{d}(t) \cos \left(\theta_{d}(t)\right) / \lambda^{3}$.

Consequently, even in tracking, the error obtained with the SMCI is less than the one obtained with the SMC, i.e., $\left(A \sin \left(\theta_{d}(t)\right)+B\right) / \lambda^{2}$.

In practical terms, this control law adds two great advantages: its state feedback works without an accelerometer sensor and there is no position error when the actuator stops.

\section{EXPERIMENTAL RESULTS}

The experimental tests were carried out by measuring the position and the two pressures $P_{p}$ and $P_{n}$. The velocity has been obtained by a numerical derivation on two sampling times.

To reduce chattering in control the discontinuous signum function is replaced by the saturation function that is linear within a boundary layer $\phi$

$$
\operatorname{sat}(\sigma / \phi)= \begin{cases}\operatorname{sign}(\sigma) & \text { if }|\sigma|>\phi \\ \sigma / \phi & \text { if }|\sigma| \leq \phi\end{cases}
$$

1) Remark: The same procedure can be followed to analyze the nonideal sliding modes (SMC and SMCI when using the 


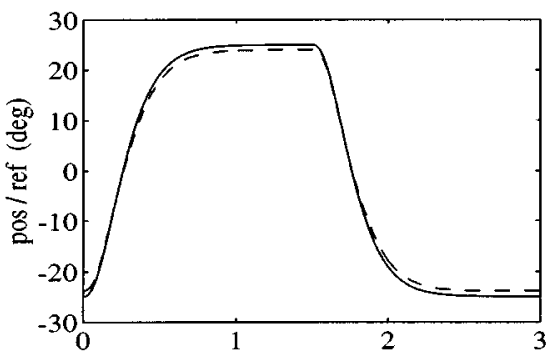

(a)

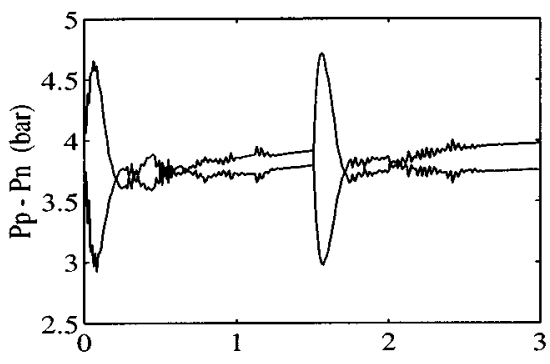

(c)

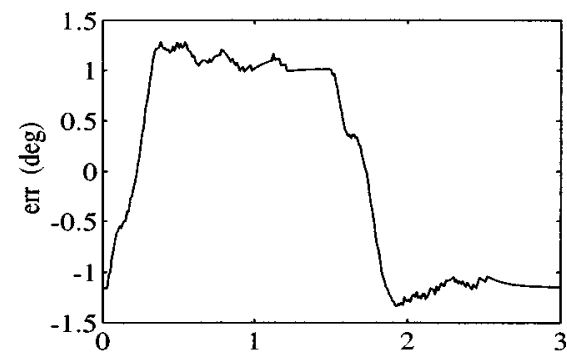

(b)

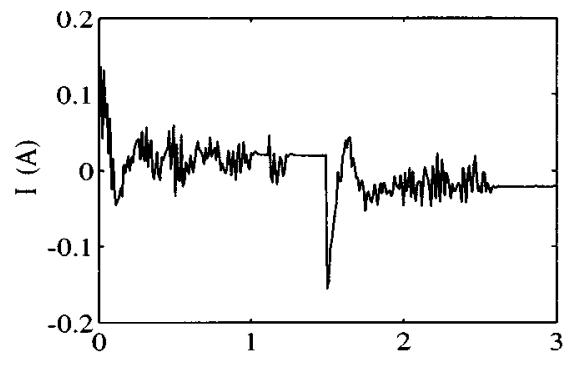

(d)

Fig. 7. SMC results near the central position.

saturation function). This is carried out by replacing the input disturbance term $\delta$ in the ideal case equations (20) and (24) by $\left(\delta+\sigma_{\max }\right)\left(\sigma_{\max }\right.$ is the bound of $\sigma$ assured by the nonideal sliding mode controller). Indeed, in [20] it has been proven that after a sufficient time, we can have the following boundedness:

$$
|\sigma| \leq \frac{\Delta_{f \max }}{W} \phi \quad \text { and } \quad|\dot{\sigma}| \leq 2 \Delta_{f \max }
$$

Thus, the sliding mode controllers (18) and (22) were implemented on a PC computer with a sampling time $T_{\text {samp }}$ fixed to $10 \mathrm{~ms}$ relative to the computing time, closed-loop response time and the velocity resolution.

To implement these control laws, three parameters are needed: the switching gain $W$, the surface coefficient $\lambda$ and the boundary layer thickness $\phi$.

$W$ is chosen [relations (19) or (23)] relative to the upper bound of $\Delta_{f}$ (obtained by upper-bounding the measured leakage mass flow rates) and is experimentally adjusted to obtain a convenient dynamics.

$$
\begin{gathered}
\Rightarrow W>\Delta_{f \max }+2 \lambda M_{\max } g l \quad \text { or } \\
W>\Delta_{f \max }+3 \lambda M_{\max } g l
\end{gathered}
$$

Therefore, because it fixes the closed loop, we have computed $\lambda$ taking into account the natural frequency of the tangent linearized model for the central position. This pulsation is about $10 \mathrm{rd} / \mathrm{s}$ and then $\lambda$ is chosen between $15 \mathrm{rd} / \mathrm{s}$ and $25 \mathrm{rd} / \mathrm{s}$.

The sliding control is mainly a switching control and it can switch a lot due to the quantization errors. To alleviate this, let $e_{\theta q}, e_{\omega q}$ and $e_{\gamma q}$, be the quantization errors of position, velocity, and acceleration, respectively. We define a quantity $\sigma_{q}$ corresponding to the value of the surface $\sigma$ (or $\sigma_{I}$ ) computed from these quantization errors.

For a 12-bit ADC and for a wide variation $-135^{\circ}$ to $+135^{\circ}$, the position resolution $e_{\theta q}$ is $0.067^{\circ}=1,16910^{-3} \mathrm{rd} / \mathrm{lsb}$.
Hence the velocity resolution $e_{\omega q}$ for our derivation method is $3.35^{\circ} / \mathrm{s} / \mathrm{sb}=0.05847 \mathrm{rd} / \mathrm{s} / \mathrm{lsb}$. The pressure resolution is 1.22 $10^{-3} \mathrm{~Pa} / \mathrm{lsb}$ and then $e_{\gamma q}=0,04 \mathrm{rd} / \mathrm{s}^{2} / \mathrm{lsb}$. Finally, for $\lambda=25$ we obtain

$$
\sigma_{q}=\lambda^{2} e_{\theta q}+2 \lambda e_{\omega q}+e_{\gamma q} \cong 4 .
$$

2) Remark: the preponderant term in computing $\sigma_{q}$ is the term in $\omega$. So, the velocity quantization error is the term the most responsible for the presence of the noise quantization in the control behavior.

The boundary layer $\phi$ is then computed as a sum of $\sigma_{q}$ and a supplementary value $\Delta \phi$ fixed experimentally: $\phi=\sigma_{q}+\Delta \phi$.

For all the tests and for both controllers SMC and SMCI, $W$, $\lambda$ and $\phi$ were imposed to: $W=750, \lambda=25$, and $\phi=20$.

The reference trajectories of position, velocity, acceleration, and jerk are obtained by means of a third-order transfer function $\left(\theta_{d} / \theta_{\text {step }}\right)=\left(1 /(1+0,1 s)^{3}\right)\left(\theta_{\text {step }}\right.$ is a step position of a given amplitude). The following graphs are referenced in degrees for easier reading.

Let us now present the experimental results. Fig. 7(a) shows the tracking curve obtained by the SMC controller (18) when applying an amplitude of $50^{\circ}$ around the central position. The dynamics is acceptable and one can see the predicted static error [Fig. 7(b)].

The control does not have much chattering [Fig. 7(d)] and the pressures $P_{p}$ and $P_{n}$ are stabilized well in the static phases [Fig. $7(\mathrm{c})]$.

More problems appear when there is tracking at the extremity of the actuator because of the high nonlinearity of the system. The obtained dynamics is bad and the static error is quite large [Fig. 8(a) and (b)].

The sliding controller with an integral surface provides better results in all cases. With the same control parameters $W, l$ and $\phi$, the tracking performances are satisfactory near the central position [Fig. 9(a)] or at the extremity [Fig. 10(a)]. As one can see 


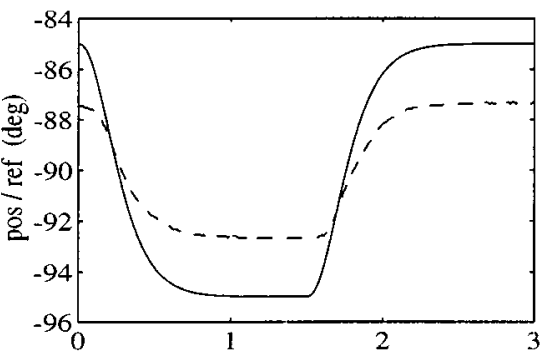

(a)

Fig. 8. SMC results at the extremity.

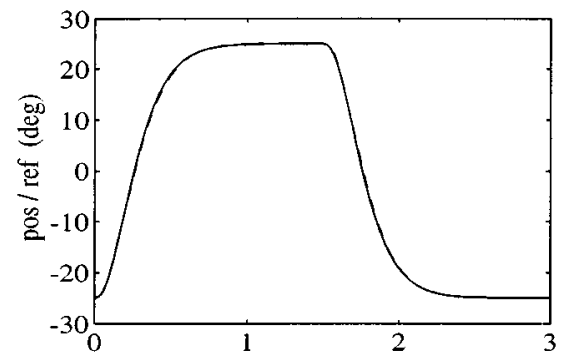

(a)

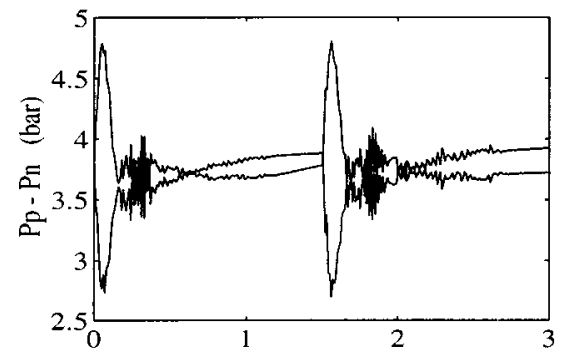

(c)

Fig. 9. SMCI results near the central position.

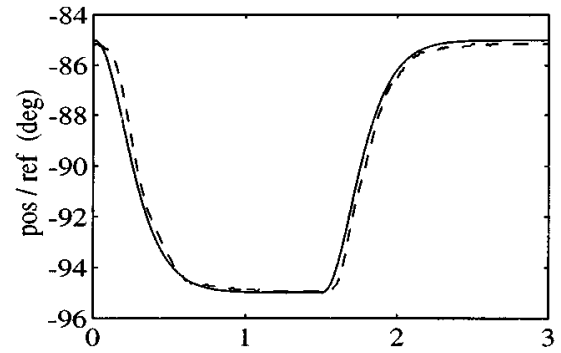

(a)

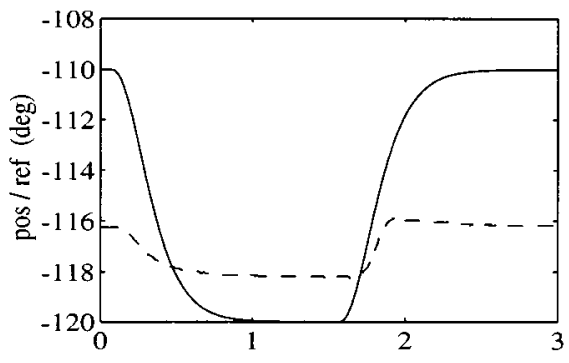

(b)

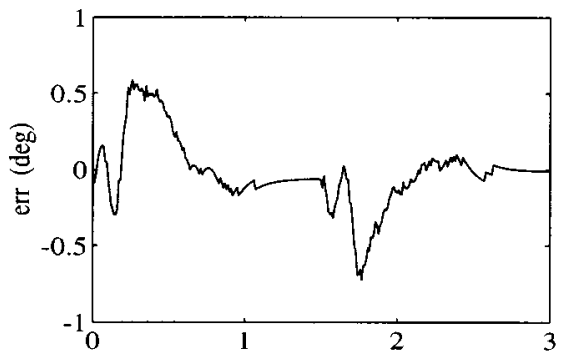

(b)

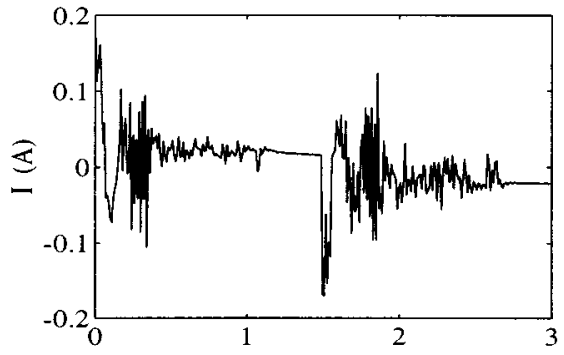

(d)

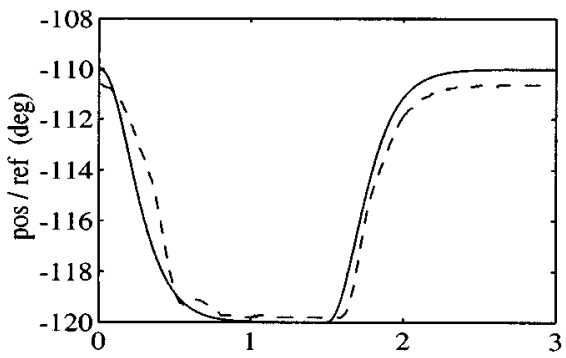

(b)

Fig. 10. SMCI results at the extremity.

the cost of this improvement is a small increase in chattering in the control current [Fig. 9(d)] but without noteworthy unpleasance for the servovalve.

\section{CONCLUSION}

As expected the sliding mode controller with an integral surface gives more useful results at all the operating points (central position and extremity). The static error has been decreased enough relative to the other controller and the dynamics is sat- isfactory especially at the extremity of the servodrive. It is important to notice the economic impact of this result with respect to the use of an accelerometer. It has been proven theoretically and experimentally that the information concerning the pressure difference may be conveniently substituted for the real acceleration.

However, the sliding mode control is not a miracle: one must always carry out a compromise between chattering and the desired performances. This compromise seemed to be held in our case. 


\section{APPENDIX}

Equation (20) may be rewritten as follows:

$$
\begin{aligned}
\ddot{e}_{\theta}+2 \lambda \dot{e}_{\theta}+\lambda^{2} e_{\theta} & =\delta\left(e_{\theta}, t\right) \\
& =\frac{M g l}{J} \sin \left(e_{\theta}+\theta_{d}(t)\right)-\frac{\Gamma_{\text {dry }}}{J} \\
& =A \sin \left(e_{\theta}+\theta_{d}(t)\right)+B / \\
A=\frac{M g l}{J}, B & =-\frac{\Gamma_{\mathrm{dry}}}{J} .
\end{aligned}
$$

We know that $\forall t \geq 0, \exists \delta_{\max } /-\delta_{\max } \leq \delta\left(e_{\theta}, t\right) \leq \delta_{\max }$, with $\delta_{\max }=A+|B|$.

Let us define a function $y$ such that $y=e_{\theta} e^{\lambda t}$.

Then $\ddot{y}=\delta\left(e_{\theta}, t\right) e^{\lambda t}$

$$
-\delta_{\max } e^{\lambda t} \leq \underbrace{\ddot{y} \leq \delta_{\max } e^{\lambda t}}_{\text {Right Ineq }}
$$

One first uses the right inequality: $\ddot{y}-\delta_{\max } e^{\lambda t} \leq 0$

By integrating this inequality we have.

$$
\begin{aligned}
\dot{y}-\frac{\delta_{\max }}{\lambda} e^{\lambda t} & \leq c_{1} \quad \text { with } \quad c_{1}=\dot{y}(0)-\frac{\delta_{\max }}{\lambda} \\
\Rightarrow \dot{y}-\frac{\delta_{\max }}{\lambda} e^{\lambda t}-c_{1} & \leq 0 .
\end{aligned}
$$

By integrating this inequality, we have

$$
\begin{aligned}
y-\frac{\delta_{\max }}{\lambda^{2}} e^{\lambda t}-c_{1} t & \leq c_{2} \quad \text { with } \quad c_{2}=y(0)-\frac{\delta_{\max }}{\lambda^{2}} \\
\Rightarrow y-\frac{\delta_{\max }}{\lambda^{2}} e^{\lambda t}-c_{1} t-c_{2} & \leq 0 .
\end{aligned}
$$

By multiplying this equation by $e^{-\lambda t}$, we obtain

$$
\begin{aligned}
e_{\theta}- & \frac{\delta_{\max }}{\lambda^{2}}\left(c_{1} t+c_{2}\right) e^{-\lambda t} \\
& \leq 0 \Rightarrow e_{\theta} \leq \frac{\delta_{\max }}{\lambda^{2}}+\underbrace{\left(c_{1} t+c_{2}\right) e^{-\lambda t}}_{\text {bounded }} .
\end{aligned}
$$

A first-order polynomial that multiply a decreasing exponential function is bounded $(t \geq 0)$.

Then, $\exists M / e_{\theta} \leq \frac{\delta_{\max }}{\lambda^{2}}$.

By considering the left inequality of $\left(^{*}\right)$ the same method may be used to prove that $\exists m / e_{\theta} \geq \frac{\delta_{\max }}{\lambda^{2}}$.

We thus prove that the position error (20) is bounded and the bounds exponentially tends (with a time constant $1 / \lambda$ ) to

$$
e_{\theta \max }=\frac{\delta_{\max }}{\lambda^{2}}=\frac{M g l+\left|T_{\mathrm{dry}}\right|}{J \lambda^{2}}
$$

The same procedure can be followed to prove the boundedness of the solution of (24) and one may find that

$$
-\frac{2 \delta_{\max }}{\lambda^{2}}+P_{2}^{\circ *} e^{-\lambda t} \leq e_{\theta} \leq \frac{2 \delta_{\max }}{\lambda^{2}}+P_{2}^{\circ} e^{-\lambda t} .
$$

$P_{2}^{\circ}, P_{2}^{\circ *}$ are two second-order polynomials.

Which exponentially tends to: $\left|e_{\theta}\right| \leq\left(2 \delta_{\max } / \lambda^{2}\right)$.

\section{ACKNOWLEDGMENT}

The authors are grateful to the referee for helpful comments and suggestions.

\section{REFERENCES}

[1] Necsulescu et al., "Low speed motion control of a mechanical system," Dyn. Contr., vol. 4, pp. 307-322, 1993.

[2] F. Boudjemaa and J. L. Abatut, "Sliding Mode-A new way to control series resonant converters," in IEEE Conf. Decision Contr., 1990.

[3] S. R. Habibi and R. J. Richards, "Computed torque and variable structure multivariable control of a hydraulic industrial robot," in Proc. Inst. Mech. Eng., vol. 205, 1991, pp. 126-140.

[4] T. Noritsugu and T. Wada, "Adaptive of variable structure control of pneumatically actuated robot," in JHPS Int. Symp. Fluid Power, Japan, Mar. 1989, pp. 591-598.

[5] D. Thomasset, E. Richard, S. Scavarda, X. F. Lin, S. Sesmat, and A. Bouhal, "Control of an electropneumatic servodrive: A state affine or a sliding approach," in Proc. IFAC World Congr., Sydney, Australia, 1993.

[6] M. Bouri, D. Thomasset, E. Richard, and S. Scavarda, "Nonlinear sliding mode control of a rotational electropneumatic servodrive," in Proc. 7th Bath Fluidpower Workshop, Sept. 1994.

[7] E. Richard and S. Scavarda, "Nonlinear control of a pneumatic servodrive," in Proc. 2nd Bath Fluidpower Workshop, Sept. 1989, pp. 59-75.

[8] A. Bouhal, "Contribution à l'étude de la commande linéaire et non linéaire des systèmes électropneumatiques," thesis (201p.), Institut National des sciences appliquées de Lyon, France, 1994.

[9] B. W. Andersen, The Analysis and Design of Pneumatic Systems. New York: Wiley, 1976.

[10] C. R. Burrows, Fluid Power Servomechanisms. London, U.K.: Van Nostrand Reinhold, 1972.

[11] F. Det, S. Scavarda, and E. Richard, "Simulation of an electropneumatic servovalve to study the feasibility of a position control loop," in Proc. 2 th Int. Conf. on Fluid Power Transmission and Control, Hangzou, China, 1989.

[12] J. Zumbrägel, "Flexible servopneumatische Greifersyteme für die Handhabungstechnik," Dissertation RTWH, Aachen, Germany, 1990.

[13] S. Sesmat and S. Scavarda, "Static characteristics of a three way electropneumatic servovalve," in 12th Aachener Fluidetechnisces Kolloquium, vol. 2, Aachen, Germany, 1996.

[14] E. Richard, "De la commande linéaire en position des systèmes électropneumatiques," Doctoral dissertation, Institut National des Sciences Appliquées de Lyon, Lyon, France, 1990.

[15] V. L. Utkin, Sliding Modes in Control Optimization. New York: Springer-Verlag, 1992.

[16] J. J. E. Slotine and W. Li, Applied Nonlinear Control. Engewlood Cliffs, NJ: Prentice-Hall, 1991.

[17] A. Isidori, Nonlinear Control Systems: An Introduction. New York: Springer-Verlag, 1989.

[18] J. J. E. Slotine, "Sliding controller design for nonlinear systems," Int. J. Contr., vol. 40, pp. 421-434, 1984.

[19] M. Bouri and D. Thomasset, "Robust position of an electropneumatic actuator via a sliding mode approach," Int. J. Robust Nonlinear Contr., to be published.

[20] M. Bouri, "Contribution à la commande non linéaire par mode de glissement. Application aux actionneurs électropneumatiques," Doctoral dissertation, Institut National des Sciences Appliquées de Lyon, Lyon, France, 1997. 